\title{
OPERATOR ESTIMATES USING THE SHARP FUNCTION
}

\author{
DOUGLAS S. KuRTZ
}

\begin{abstract}
Let $f^{\#}$ be the sharp function introduced by Fefferman and Stein. Suppose that $T$ and $U$ are operators acting on the space of Schwartz functions which satisfy the pointwise estimate $(T f)^{\#}(x) \leq A|U f(x)|$. Then, on the $L^{p}$ spaces, the operator norm of $T$ divided by the operator norm of $U$ is bounded by a constant times $p$. This result allows us to obtain the best possible rate of growth estimate, as $p \rightarrow \infty$, on the norms of singular integrals, multipliers, and pseudo-differential operators. These estimates remain valid on weighted $L^{p}$ spaces defined by an $A_{\infty}$ weight.
\end{abstract}

Introduction. Let $\mathscr{S}$ be the space of Schwartz testing functions and suppose that $T$ and $U$ are two operators acting on $\mathscr{S}$. Assume that for all $f \in \mathscr{S}$ we have the pointwise inequality

$$
(T f)^{\#}(x) \leq A|U f(x)|,
$$

where $g^{\#}$ is the Fefferman and Stein sharp function. It is well known that (1) implies the $L^{p}$ inequality $\|T f\|_{p} \leq C(p)\|U f\|_{p}, 1<p<\infty$, where the constant $C(p)$ grows exponentially in $p$. Moreover, these norms can be replaced by a weighted norm as long as the weight satisfies the $A_{\infty}$ condition.

In fact, inequality (1) implies a much stronger result. We show that as long as $T f$ satisfies mild growth conditions for all $f \in \mathscr{S}$, the constant $C(p)$ grows linearly in $p$. This result is then used to prove best possible norm estimates for a large class of singular integral and multiplier operators.

The proof of the main theorem is based on a better understanding of the distribution function of the sharp function. The standard norm estimate for the sharp function is a consequence of what is referred to as a "good- $\lambda$ inequality." In Lemma 1 we improve the good- $\lambda$ inequality by showing

(2) $w\left(\left\{x \in \mathbb{R}^{n}:|f(x)|>B f^{\#}(x)+\lambda\right\}\right) \leq \varepsilon w\left(\left\{x \in \mathbb{R}^{n}:|f(x)|>\lambda\right\}\right)$. 
Using this inequality and considering non-increasing rearrangements of $f$ and $f^{\#}$ we are able to derive

THEOREM 1. Let $w \in A_{\infty}$. There is a constant $C$, depending on $w$ and $n$ so that if $f$ is locally integrable and $w\left(\left\{x \in \mathbb{R}^{n}:|f(x)|>\lambda\right\}\right)<$ $+\infty$ for all $\lambda>0$, then

$$
\|f\|_{p, w} \leq C \cdot p\left\|f^{\#}\right\|_{p, w}, \quad 1 \leq p<\infty .
$$

Thus, we get that the ratio $\|f\|_{p, w} /\left\|f^{\#}\right\|_{p, w}$ grows linearly in $p$. This estimate allows us to obtain relatively sharp operator estimates from an inequality like (1).

The paper is divided into three sections. In the first section, we consider the sharp function itself and prove our basic results. The second section derives the consequences of the estimate (1). Finally, in $\S 3$, we list known cases of the estimate (1) and discuss some situations where it implies best possible estimates.

1. Results for the sharp function. Let $g$ be a locally integrable function on $\mathbb{R}^{n}$. We define the sharp function of $g$ by

$$
g^{\#}(x)=\sup _{x \in Q} \frac{1}{|Q|} \int_{Q}\left|g(y)-g_{Q}\right| d y,
$$

where $g_{Q}=(1 / Q) \int_{Q} g(y) d y$. The sharp function was introduced by C. Fefferman and E. M. Stein in [13]. Let $w$ be a non-negative, locally integrable function. For any Lebesgue measurable set $E$, let $w(E)=\int_{E} w(x) d x$. We say that $w$ satisfies the $A_{\infty}$ condition if given $\varepsilon>0$ there is a $\delta>0$ so that for any cube $Q \subset \mathbb{R}^{n}$ and any measurable set $E \subset Q, m(E)<\delta m(Q)$ implies that $w(E)<\varepsilon w(Q)$.

All of our results are based on the following lemma. Its statement and proof generalizes those of Theorem 4.1 of [2], as well as the original result proved by Fefferman and Stein.

Lemma 2. Let $w \in A_{\infty}$. Given $\varepsilon>0$, there is a constant $B$, depending on $w, \varepsilon$, and $n$, so that for all locally integrable functions $f$ and $\lambda>0$,

$$
\begin{aligned}
w\left(\left\{x \in \mathbb{R}^{n}:|f(x)|>B f^{\#}(x)+\lambda\right\}\right) \\
\leq \varepsilon w\left(\left\{x \in \mathbb{R}^{n}:|f(x)|>\lambda\right\}\right) .
\end{aligned}
$$

Proof. Fix $\varepsilon>0$. Let $E_{\lambda}=\left\{x \in \mathbb{R}^{n}:|f(x)|>\lambda\right\}$. Without loss of generality, we may assume that $0<w\left(E_{\lambda}\right)<\infty$, and hence that $f$ is 
not identically constant. It follows that $f^{\#}(x)>0$ for all $x \in \mathbb{R}^{n}$ and, moreover, that ess inf $Q f^{\#}>0$ for any cube $Q$.

Let $G$ be an open set containing $E_{\lambda}$ such that $w(G) \leq 2 w\left(E_{\lambda}\right)$. By Lemma 3.1 of [2], we can cover $G$ by a sequence of nonoverlapping cubes $\left\{Q_{k}\right\}$ so that $m\left(Q_{k}\right) \leq 2 m\left(Q_{k} \backslash G\right)$ and $\sum w\left(Q_{k}\right) \leq C w(G)$, where $C$ depends only on $w, \varepsilon$, and $n$.

Fix $Q \in\left\{Q_{k}\right\}$. The argument used to prove Theorem 4.1 [2] shows that $\left|f_{Q}\right| \leq 2 f^{\#}(x)+\lambda$ for all $x \in Q$. Set $I=\operatorname{essinf}_{Q} f^{\#}>0$. Let $\mu=\frac{1}{\delta} I+\left|f_{Q}\right|$ and $E=E_{\mu} \cap Q$, with $\delta$ to be chosen. Again, arguing as in Theorem 4.1, $\left(\mu-\left|f_{Q}\right|\right) m(E) \leq m(Q) f^{\#}(x)$ for all $x \in Q$. This implies that $\frac{1}{\delta} \operatorname{Im}(E) \leq I m(Q)$, so that $m(E) \leq \delta m(Q)$. Therefore, if we set $B=2+\frac{1}{\delta}$, we have

$$
\begin{aligned}
m(\{x \in Q: \mid & \left.\left.f(x) \mid>B f^{\#}(x)+\lambda\right\}\right) \\
& =m\left(\left\{x \in Q:|f(x)|>\frac{1}{\delta} f^{\#}(x)+\left[2 f^{\#}(x)+\lambda\right]\right\}\right) \\
& \leq m\left(\left\{x \in Q:|f(x)|>\frac{1}{\delta} I+\left|f_{Q}\right|\right\}\right)=m(E)<\delta m(Q) .
\end{aligned}
$$

Now, choose $\delta$ corresponding to $\varepsilon / 2 C$ in the definition of $A_{\infty}$. Then,

$$
w\left(\left\{x \in Q:|f(x)|>B f^{\#}(x)+\lambda\right\}\right)<(\varepsilon / 2 C) w(Q) .
$$

Since this is true for all cubes in $\left\{Q_{k}\right\}$ and the cubes are disjoint,

$$
\begin{aligned}
w\left(\left\{x \in \mathbb{R}^{n}:\right.\right. & \left.\left.|f(x)|>B f^{\#}(x)+\lambda\right\}\right) \\
& <(\varepsilon / 2 C) \sum w(Q) \leq(\varepsilon / 2) w(G) \leq \varepsilon w\left(E_{\lambda}\right) .
\end{aligned}
$$

Define the generalized Hardy-Littlewood maximal function of $g$ by

$$
M_{r} g(x)=\sup \left(\frac{1}{|Q|} \int_{Q}|g(y)|^{r} d y\right)^{1 / r}, \quad 1 \leq r<\infty,
$$

where the supremum is taken over all cubes $Q$ which contain $x$. The lemma is also true with $|f|$ replaced by $M f=M_{1} f$ in the conclusion. This improves the estimate of Fefferman and Stein [13].

Let $q>0$ and define the sharp function $g_{q}^{\#}$ by

$$
g_{q}^{\#}(x)=\sup _{x \in Q}\left(\frac{1}{|Q|} \int_{Q}\left|g(y)-g_{Q}\right|^{q} d y\right)^{1 / q} .
$$

By Hölder's inequality, for $q \geq 1, g^{\#}(x) \leq g_{q}^{\#}(x)$ so that Lemma 2 is valid with $f^{\#}$ replaced by $f_{q}^{\#}$. Further, for $0<q<1$, we can use the argument above, with the minor changes that $\left|f_{Q}\right| \leq 2^{1 / q} f_{q}^{\#}(x)+\lambda$ 
and $m(E) \leq \delta^{1 / q} m(Q)$. This generalization is useful for one of the applications.

The proof of Theorem 1 is now fairly straightforward. (See, for example, [1].) Define the non-increasing rearrangement function of $f$ with respect to $w$ by

$$
f_{w}^{*}(t)=\inf \left\{\lambda>0: w\left(\left\{x \in \mathbb{R}^{n}:|f(x)|>\lambda\right\}\right) \leq t\right\} .
$$

Let $\lambda=f_{w}^{*}(2 t)$ and $\varepsilon=1 / 4$ in Lemma 2 . By the definition of the non-increasing rearrangement,

$$
f_{w}^{*}(t) \leq C\left(f^{\#}\right)_{w}^{*}\left(\frac{t}{2}\right)+f_{w}^{*}(2 t)
$$

Iterating this inequality, we have

$$
f_{w}^{*}(t) \leq C \int_{t / 4}^{\infty}\left(f^{\#}\right)_{w}^{*}(s) d s / s+\lim _{s \rightarrow \infty} f_{w}^{*}(s),
$$

where the limit term is zero by the assumptions on $f$. The theorem now follows by an application of Hardy's inequality.

2. Pointwise estimates and good- $\lambda$ inequalities. Let $T$ and $U$ be two operators acting on $\mathscr{S}$. Say that $T$ and $U$ satisfy condition $\mathrm{S}$ with respect to $w$ if for all $f \in \mathscr{S}$ :

(i) $T f$ is locally integrable,

(ii) $w\left(\left\{x \in \mathbb{R}^{n}:|T f(x)|>\lambda\right\}\right)<+\infty$, for all $\lambda>0$,

(iii) $(T f)^{\#}(x) \leq A|U f(x)|$ a.e.

From Theorem 1 we have

THEOREM 3. Let $w \in A_{\infty}$ and suppose that $T$ and $U$ satisfy condition $\mathrm{S}$ with respect to $w$. There is a constant $C$ depending on $w, A$, and $n$ so that

$$
\|T f\|_{p, w} \leq C \cdot p\|U f\|_{p, w}, \quad 1<p<\infty .
$$

However, from Lemma 2 we can derive more. If we set $C=A B$, then

$$
\begin{aligned}
w(\{x:|T f(x)|>C|U f(x)|+\lambda\}) \\
\quad \leq w\left(\left\{x:|T f(x)|>B(T f)^{\#}(x)+\lambda\right\}\right) \\
\quad \leq \varepsilon w(\{x:|T f(x)|>\lambda\}) .
\end{aligned}
$$

Thus, we have

LemMa 4. Let $w \in A_{\infty}$ and suppose that $T$ and $U$ satisfy condition $\mathrm{S}$ with respect to $w$. Given $\varepsilon>0$ there is a constant $C$ depending on 
$w, \varepsilon, A$, and $n$ so that

$$
w(\{x:|T f(x)|>C|U f(x)|+\lambda\}) \leq \varepsilon w(\{w:|T f(x)|>\lambda\})
$$

for all $f \in \mathscr{S}$ and $\lambda>0$.

In fact, the lemma is true as long as $T$ and $U$ satisfy (i) and (iii). We observe that this "good- $\lambda$ " type inequality is actually stronger than the more standard estimate of this type. Setting $C=1 / \delta$, we get an estimate of the form

$$
w(\{x:|T f(x)|>2 \lambda \text { and }|U f(x)| \leq \delta \lambda\}) \leq \varepsilon w(\{x:|T f(x)|>\lambda\}) .
$$

Additionally, Lemma 4 implies Theorem 3 while this last inequality does not.

By condition (ii), $(T f)^{*}(t) \rightarrow 0$ as $t \rightarrow \infty$. Therefore, arguing as in the proof of Theorem 1, Lemma 4 implies

Lemma 5. Let $w \in A_{\infty}$ and suppose that $T$ and $U$ satisfy condition $\mathrm{S}$ with respect to $w$. Then, there is a constant $C$ depending on $w, A$, and $n$ so that for $f \in \mathscr{S}$ and $t>0$,

$$
(T f)_{w}^{*}(t) \leq C \int_{t / 4}^{\infty}(U f)_{w}^{*}(s) \frac{d s}{s} .
$$

In general, our interest is to derive properties of $T$ from ones satisfied by $U$. Suppose that $U$ satisfies weak-type $(r, r), r \geq 1$, and strong-type $(\infty, \infty)$ conditions with respect to a fixed weight $w$. It follows that the norm of $U$ is bounded for large $p$, so that Theorem 3 implies

Corollary 6. Let $w \in A_{\infty}$ and suppose that $T$ and $U$ satisfy conditon $\mathrm{S}$ with respect to $w$. If $U$ satisfies weak-type $(r, r), r \geq 1$, and strong-type $(\infty, \infty)$ conditions with respect to $w$, then there are constants $p_{0}>r$ and $C$, depending on $w$ and $A$, so that

$$
\|T f\|_{p, w} \leq C \cdot p\|f\|_{p, w}, \quad p_{0} \leq p<\infty .
$$

Further, by Calderón's theorem [5],

$$
(U f)_{w}^{*}(t) \leq C t^{-1 / r} \int_{0}^{t} s^{1 / r} f_{w}^{*}(s) \frac{d s}{s} .
$$


Using this estimate in the conclusion of Lemma 5 yields

Corollary 7. Let $w \in A_{\infty}$ and suppose that $T$ and $U$ satisfy condition $\mathrm{S}$ with respect to $w$. If $U$ satisfies weak-type $(r, r), r \geq 1$, and strong-type $(\infty, \infty)$ conditions with respect to $w$, then there is a constant $C$ so that for $f \in \mathscr{S}$ and $t>0$

$$
(T f)_{w}^{*}(t) \leq C\left\{t^{-1 / r} \int_{0}^{t} s^{1 / r} f_{w}^{*}(s) \frac{d s}{s}+\int_{t}^{\infty} f_{w}^{*}(s) \frac{d s}{s}\right\} .
$$

Inequalities such as this have been studied by Calderón [5] when the operator $T$ satisfies various weak-type conditions and later generalized by Bennett and Rudnick [4]. This result shows that they follow from properties of $U$.

Analogs of Corollaries 6 and 7 follow when $U$ satisfies two weaktype conditions or weak-type $(p, q)$ conditions. Since most of our applications use majorants which are bounded on $L^{\infty}$, we do not state these corollaries in such generalities.

3. Applications. There are many applications of the estimate $(T f)^{\#}(x) \leq A|U f(x)|$. It follows from Theorem 1 that in some cases this inequality can be used to prove sharp estimates on the rate of growth of the norm of the operator $T$.

This estimate was first used by Córdoba and Fefferman [12] to study singular integral operators with kernels satisfying a gradient condition. It was later generalized by Kurtz and Wheeden [16] to more general singular integral operators.

Let $\Sigma=\Sigma_{n-1}=\left\{x \in \mathbb{R}^{n}:|x|=1\right\}$. Given a rotation $\rho$, set $|\rho|=$ $\sup _{x \in \Sigma}|x-\rho x|$.

Definition 8 . Let $\Omega$ be defined on $\Sigma$ and satisfy $\int_{\Sigma} \Omega d \sigma=0$. For $1 \leq r \leq \infty$, define the $L^{r}$-modulus of continuity of $\Omega$ on $\Sigma$ by

$$
\omega_{r}(\delta)=\sup _{|\rho| \leq \delta}\left(\int_{\Sigma}|\Omega(x)-\Omega(\rho x)|^{r} d \sigma(x)\right)^{1 / r},
$$

with the obvious modification when $r=\infty$. We say that $\Omega$ satisfies the $L^{r}$-Dini condition of $\Omega \in L^{r}(\Sigma)$ and $\int_{0}^{1}\left(\omega_{r}(\delta) / \delta\right) d \delta<+\infty$.

Suppose $K(x)=\Omega(x) /|x|^{n}$, where $\Omega \in L^{r}$-Dini for some $r>1$. Define the singular integral operator $T$ by

$$
T f(x)=\lim _{\varepsilon \downarrow 0} \int_{\{|y|>\varepsilon\}} K(y) f(x-y) d y .
$$


As shown in [16], if $\Omega \in L^{r}$-Dini, then $(T f)^{\#}(x) \leq A M_{r^{\prime}} f(x)$. Since $M_{r^{\prime}}$ is weak-type $\left(r^{\prime}, r^{\prime}\right)$ and strong-type $(\infty, \infty)$, from Theorem 6 we get

Theorem 9. Let $w \in A_{\infty}$ and $1<r \leq \infty$. Suppose that $\Omega \in L^{r}$ Dini. Then, there exist constants $C$ and $p_{0}$, depending on $w, r, \Omega$, and $n$, such that

$$
\|T f\|_{p, w} \leq C \cdot p\|f\|_{p, w}, \quad p_{0} \leq p<\infty .
$$

This result had previously been proved in [1] for the maximal singular integral operator associated to $T$. Since the kernel of the Hilbert transform satisfies all these Dini conditions, we see that this is the best possible estimate on the rate of growth of the norm of $T$, even in the unweighted case.

For $f \in \mathscr{S}$, define the Fourier transform of $f$ by

$$
\hat{f}(\xi)=\int_{\mathbb{R}^{n}} e^{-2 \pi i y \cdot \xi} f(y) d y .
$$

Given a bounded function $m(x)$ on $\mathbb{R}^{n}$, define the multiplier operator $T=T_{m}$ by $(T f)^{\wedge}(x)=m(x) \hat{f}(x), f \in \mathscr{S}$. Let $\beta=\left(\beta_{1}, \ldots, \beta_{n}\right)$ be a multi-index of non-negative integers and set $|\beta|=\beta_{1}+\cdots+\beta_{n}$. Consider multipliers, $m$, which satisfy the following definition.

Definition 10. Let $1 \leq s<\infty$ and $l \in \mathbb{N}$. We say $m \in M(s, l)$ if there is a constant $B$ such that $|m(x)| \leq B$ and

$$
\left(R^{s|\beta|-n} \int_{\{x: R<|x|<2 R\}}\left|D^{\beta} m(x)\right|^{s} d x\right)^{1 / s} \leq B, \quad R>0,|\beta| \leq l .
$$

For $l>n / 2, M(2, l)$ is the usual Hörmander condition. The condition above was studied in [16] where it was shown that for $1<s \leq 2$, $n / s<l \leq n$, and $r>n / l$, there is constant $A$ so that $\left(T_{N} f\right)^{\#}(x) \leq$ $A M_{r} f(x)$. Here, $T_{N}$ is defined by a smooth cutoff of $m, T_{N}$ converges to $T$ as $N \rightarrow \infty$, and the constant $A$ is independent of $N$. Thus, we get the analog of Theorem 9 for these multiplier operators.

Theorem 11. Let $w \in A_{\infty}$. Let $1<s \leq 2, n / s<l \leq n$, and $m \in$ $M(s, l)$. Then there exist constants $C$ and $p_{0}$, depending on $w, B, s, l$, and $n$, such that

$$
\|T f\|_{p, w} \leq C \cdot p\|f\|_{p, w}, \quad p_{0} \leq p<\infty .
$$


Again, the linear growth of the norm of the operator is sharp, as can be seen by considering the multiplier associated to the Hilbert transform.

This technique can also be used to study square functions, although in the unweighted case this does not yield the best possible estimates. Let $f$ be defined on $\mathbb{R}^{n}$. For $x \in \mathbb{R}^{n}$ and $t>0$, let $f(x, t)$ be the Poisson integral of $f$ and $\nabla f$ the gradient of $f(x, t)$. The LittlewoodPaley functions of $f$ are defined as

$$
\begin{aligned}
& g(f)(x)=\left(\int_{0}^{\infty} t|\nabla f(x, t)|^{2} d t\right)^{1 / 2}, \\
& S(f)(x)=\left(\iint_{\left\{(z, t) \in \mathbf{R}_{+}^{n+1}:|z-x|<t\right\}} t^{1-n}|\nabla f(z, t)|^{2} d z d t\right)^{1 / 2},
\end{aligned}
$$

and

$$
\begin{aligned}
g_{\lambda}^{*}(f) & (x) \\
= & \left(\iint_{\mathbf{R}_{+}^{n_{1}}}\left(\frac{t}{t+|x-z|}\right)^{\lambda n} t^{1-n}|\nabla f(z, t)|^{2} d z d t\right)^{1 / 2}, \quad 1<\lambda .
\end{aligned}
$$

These operators can be generalized to involve less standard kernels as follows. Let $\varphi$ be a Schwartz function with integral mean value zero, $\int \varphi=0$. For $t>0$, set $\varphi_{t}(x)=t^{-n} \varphi(x / t)$. We define the analogs of the Littlewood-Paley operators by:

$$
\begin{aligned}
& g[\varphi](f)(x)=\left(\int_{0}^{\infty}\left|\varphi_{t} * f(x)\right|^{2} t^{-1} d t\right)^{1 / 2}, \\
& S[\varphi](f)(x)=\left(\iint_{\left\{(z, t) \in \mathrm{R}_{+}^{n+1}:|z-x|<t\right\}}\left|\varphi_{t} * f(z)\right|^{2} t^{-1-n} d z d t\right)^{1 / 2},
\end{aligned}
$$

and

$$
\begin{aligned}
& g_{\lambda}^{*}[\varphi](f)(x) \\
& \quad=\left(\iint_{\mathbf{R}_{+}^{n+1}}\left(\frac{t}{t+|x-z|}\right)^{\lambda n} t^{-1-n}\left|\varphi_{t} * f(z)\right|^{2} d z d t\right)^{1 / 2}, \quad 1<\lambda .
\end{aligned}
$$

Each of these operators can be shown to satisfy an estimate $(T f)^{\#}(x) \leq$ $A M_{r} f(x)$, where $r>1$ depends on the operator and can always be taken to be 2 .

Let $w \in A_{\infty}$. It follows that all of these operators have norms that grow at most linearly in $p$ on the weighted $L^{p}$ spaces. When we consider unweighted spaces, the norms of these operators are known 
to grow like $\sqrt{p}$ (as long as $\lambda \geq(n+1) / n$ ). See for example $[3,6,10]$. For the weighted spaces, such an estimate is unknown.

Let $\sigma(x, \xi)$ be a function defined on $\mathbb{R}^{n} \times \mathbb{R}^{n}$, and define the operator $A$ by $A f(x)=\int_{\mathbb{R}^{n}} e^{2 \pi i x \cdot \xi} \sigma(x, \xi) \hat{f}(\xi) d \xi$. We call $A$ a pseudodifferential operator with symbol $\sigma$. We say that $\sigma \in S_{\rho, \delta}^{m}$ if for every $x, \zeta \in \mathbb{R}^{n}$,

$$
\left|\frac{\partial^{\alpha}}{\partial x^{\alpha}} \frac{\partial^{\beta}}{\partial \xi^{\beta}} \sigma(x, \xi)\right| \leq c_{\alpha, \beta}(1+|\xi|)^{m-\rho|\beta|+\delta|\alpha|},
$$

and call $\sigma$ a symbol of order $m$ if $\sigma \in S_{1,0}^{m}$. In [17], Miller showed that for a symbol $\sigma$ of order 0 and $1<r<\infty$, there is a constant $C$ such that $(A f)^{\#}(x) \leq C M_{r} f(x)$. He used this to study pseudo-differential operators with symbols of order 0 on weighted $L^{p}$ spaces and ones with symbols of order $m$ on weighted Sobolev spaces. Since then, many authors have used this technique to study pseudo-differential operators for symbols in various classes, $S_{\rho, \delta}^{m}$. Note that when $\sigma$ is independent of $x, A$ is a multiplier operator. One sees that this technique yields best possible estimates for pseudo-differential operators in some cases since the multiplier associated with the Hilbert transform is a symbol in $S_{\rho, \delta}^{m}$ for all $m, \rho$, and $\delta$, with $m \geq 0$.

Related to the operators above are the convolution operators with oscillating kernels. For $t \in \mathbb{R}$, these operators are defined by the kernels $K_{a, b+i y}(t)=\exp \left(i|t|^{a}\right)(1+|t|)^{-b-i y}$. For $0<a \neq 1$ and $1<r<\infty$, there is a constant $C$ so that $\left(K_{a, 1+i y} * f\right)^{\#}(x) \leq C(1+|y|) M_{r} f(x)$. (See [8].)

Let $0<\alpha<n$. Suppose $T$ is a convolution operator with a kernel $K$ which satisfies

(i) $\left|\left\{x \in \mathbb{R}^{n}:|K(x)|>\lambda\right\}\right| \leq C \lambda^{-n /(n-\alpha)}$

(ii) $|K(x-y)-K(x)| \leq C|y| /|x|^{n+1-\alpha},|x|>2|y|$

or a multiplier operator with a multiplier $m$ which satisfies $|m(x)| \leq$ $B|x|^{-\alpha}$ and

$$
\left(R^{s|\beta|+s \alpha-n} \int_{\{x: R<|x|<2 R\}}\left|D^{\beta} m(x)\right|^{s} d x\right)^{1 / s} \leq B, \quad R>0,
$$

for some $s, 1<s \leq 2$, and all $|\beta| \leq n / s$. For $1 \leq r<n / \alpha$, define $M_{\alpha, r}$ by

$$
M_{\alpha, r} f(x)=\sup _{Q}\left(|Q|^{(\alpha r / n)-1} \int_{Q}|f(y)|^{r} d y\right)^{1 / r} .
$$


As shown in [15], we have $(T f)^{\#}(x) \leq A M_{\alpha, r} f(x)$, where $r>1$ and depends on the conditions satisfied by $K$ or $m$. (For the multiplier operators, we must use truncations of $m$ as mentioned above.) This applies to the fractional integral operators

$$
\begin{aligned}
& I_{\alpha} f(x)=\frac{1}{\delta(\alpha)} \int_{\mathbb{R}^{n}} f(y)|x-y|^{\alpha-n} d y, \\
& \quad \text { with } \delta(\alpha)=\Pi^{n / 2} 2^{\alpha} \Gamma\left(\frac{n}{2}\right) / \Gamma\left(\frac{n}{2}-\frac{\alpha}{2}\right) .
\end{aligned}
$$

Thus, this can be used to study operators from $L^{p}$ into $L^{q}$ with $q$ different than $p$.

The last application involves some recent work on operators with kernels which satisfy properties defined in terms of an $A_{1}$ weight [11]. Such operators $T$ satisfy the estimate $(T f)_{q}^{\#}(x) \leq C \lambda(f)(x)$ for some $q>0$. Here $\lambda=\lambda_{w}^{\beta, \varepsilon}$ is an infinite sum of $L^{1}$ averages of $f$ times averages of the weight, $w$.

\section{REFERENCES}

[1] R. J. Bagby and D. S. Kurtz, A rearranged good- $\lambda$ inequality, Trans. Amer. Math. Soc., 293 (1986), 71-81.

[2] Covering lemmas and the sharp function, Proc. Amer. Math. Soc., 93 (1985), 291-296.

[3] R. Bañuelos, Brownian motion and area functions, (preprint).

[4] C. Bennett and K. Rudnick, On Lorentz-Zygmund spaces, Dissertationes Math., 175 (1980), 1-67.

[5] A. P. Calderon, Spaces between $L^{1}$ and $L^{\infty}$ and the theorem of Marcinkiewicz, Studia Math., 26 (1966), 273-299.

[6] S. Y. A. Chang, J. Wilson, and T. Wolff, Some weighted norm inequalities concerning the Schrödinger operators, Comment. Math. Helv., 60 (1985), 217-246.

[7] S. Chanillo, Weighted norm inequalities for strongly singular convolution operators, Trans. Amer. Math. Soc., 281 (1984), 77-107.

[8] S. Chanillo, D. S. Kurtz, and G. Sampson, Weighted $L^{p}$ estimates for oscillating kernels, Ark. Math., 21 (1983), 233-257.

[9] S. Chanillo and A. Torchinsky, Sharp function and weighted $L^{p}$ estimates for a class of pseudo-differential operators, Ark. Math., 24 (1986), 1-25.

[10] S. Chanillo and R. L. Wheeden, Some norm inequalities for the area integral, I. U. Math. J., 36, No. 2 (1987), 277-294.

[11] R. Coifman, G. David, Y. Meyer, and S. Semmes, $\omega$-Calderón-Zygmund operators, (preprint).

[12] A. Cordoba and C. Fefferman, A weighted norm inequality for singular integrals, Studia Math., 57 (1976), 97-101.

[13] C. Fefferman and E. M. Stein, $H^{p}$ spaces of several variables, Acta Math., 129 (1972), 137-193. 
[14] J. Garcia-Cuerva and J. L. Rubio de Francia, Weighted Norm Inequalities and Related Topics, North-Holland, Amsterdam, 1985.

[15] D. S. Kurtz, Sharp function estimates for fractional integrals and related operators, (preprint).

[16] D. S. Kurtz and R. L. Wheeden, Resultls on weighted norm inequalities for multipliers, Trans. Amer. Math. Soc., 255 (1979), 343-362.

[17] N. Miller, Weighted Sobolev spaces and pseudo-differential operators with smooth symbols, Trans. Amer. Math. Soc., 269 (1982), 91-109.

[18] A. Miyachi and K. Yabuta, Sharp function estimates for pseudo-differential operators of class $S_{\rho, \delta}^{m}$, Bull. Fac. Sci., Ibaraki Univ., Math., No. 19 (1987), 15-30.

[19] J. L. Rubio de Francia, F. J. Ruiz and J. L. Torrea, Calderón-Zygmund theory for operator-valued kernels, Adv. in Math., 62 (1986), 7-48.

Received March 20, 1988. The author acknowledges the support of the Centre for Mathematical Analysis, Canberra, Australia, and Minigrant \#RC-87-28 from the College of Arts and Sciences, New Mexico State University, in the preparation of this material.

New Mexico State University

LAS CRUCES, NM 88003-0001

AND

Australian National University

GPO BOX 4

Canberra ACT 2601, Australia 
